\title{
Staphylococcal Scalded Skin Syndrome in a Neonate
}

Sir,

Staphylococcal scalded skin syndrome (SSSS) manifests acute exfoliation of skin following a staphylococcal infection. We report a case of SSSS with pneumonia, an unusual combination in neonates. A full term, $3 \mathrm{~kg}$, boy presented on the $18^{\text {th }}$ day of life with erythematous, bullous skin lesions over face, back \& extremities. Baby was irritable on admission. Perinatal period was uneventful. There was no family history of similar skin lesions. On admission, Nikolsky sign was positive. No systemic involvement was noted. Blood culture, gastric aspirate and pharyngeal swab cultures were done. With clinical suspicion of SSSS (Fig. 1) intravenous amoxicillin and clavulinic acid combination alongwith amikacin was initiated. Appropriate skin care was initiated. Desquamation stopped within 24 hours. On the second day, scaling lesions spread all over body. On the third day baby developed respiratory distress with bilateral crepitations. Chest roentgenogram showed right lung consolidation. Intravenous vancomycin was initiated. Respiratory distress settled over next two days. Blood culture was sterile. Pharyngeal swab and gastric aspirate cultures were positive for Staphylococcus aureus. Skin lesions healed without scarring.

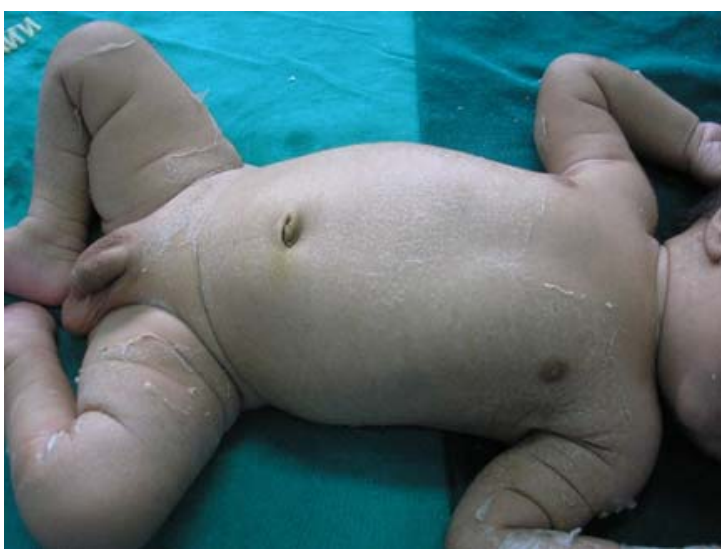

Fig. 1. Exfoliated skin lesions.

Exfoliative skin diseases of neonates are relatively rare ${ }^{1}$ although cases of fetal congenital SSSS are reported. ${ }^{2}$ SSSS is a toxin mediated staphylococcal infection. Staphylococcal ETA and ETB are secreted mainly from Phase II staphylococci. The split in the granular layer is attributable to binding of ETA or ETB to desmoglein within desmosomes and to keratohyalin granules and the granular layers. ${ }^{3}$ These toxins induce proteolysis by trypsin like serine proteases. ${ }^{4}$ ETA exhibits superantigen activities.

The complications of SSSS include fluid loss, septic arthritis, cellulitis, pneumonia, sepsis, and osteomyelitis. These are important causes of mortality. Blood culture may be sterile as in our patient. Skin culture and skin biopsy is useful. In our case parents refused consent for skin biopsy. Pharyngeal swab and gastric aspirate are helpful to isolate staphylococci secreting ETA and ETB. ${ }^{5}$

High index of suspicion and prompt intravenous antibiotics help in good outcome. Desquamation usually ceases in 24 hours of antibiotic therapy. ${ }^{1}$ Skin lesions resolve slowly over next few days without scarring. In VLBW babies, SSSS can be life threatening and it can cause serious outbreaks in nursery.

At present antibiotics form the main pillar of treatment, but with a growing concern of resistant strains of staphylococci ${ }^{6}$ Anti-ETA and Anti-ETB antibodies may appear to be the form of future therapeutics.

Sandeep Kadam, Amit Tagare, Jayant Deodhar, Yashwant Tawade ${ }^{1}$ and Anand Pandit Division of Neonatology, Department of Pediatrics ${ }^{1}$ Department of Dermatology KEM Hospital, Rasta Peth, Pune 411011, India E-mail:drsandeepkadam@yahoo.com

\section{REFERENCES}

1. Coleman JC, Dobson NR. Diagnostic dilemma: extremely low birth weight baby with staphylococcal scalded skin syndrome or toxic epidermal necrolysis. J Perinatol 2006; 26 : 714-716.

2. Haveman LM, Fleet A, de Vries LS, Gerards LJ. Congenital staphylococcal scalded skin syndrome in premature infant. Acta Pediatr 2004; 93 : 1661-1662.

3. Smith TP, John DA, Bailey CJ. The binding of epidermolytic toxin from staphylococcus aureus to mouse epidermal tissue. Histo chem J 1987; 19: 137-149.

4. Rago JV, Vath GM, Bohach GA, Ohlendorf DH, Schrlivert PM. Mutational analysis of superantigen staphylococcal exfoliative toxin A (ETA). J Immunol 2000; 164 : 2207-2213.

5. Rieger - Fackeldey E, Plano LR, Kramet A, Schulze A. Staphylococcal scalded skin syndrome related to an exfoliative toxin A and B producing strain in preterm infants. Eur J Pediatr 2002; 161 : 649-652.

6. Acland KM, Darvay A, Griffin C, Aali SA, Russell Jones R. Staphylococcal scalded skin syndrome in an adult associated with methicillin resistant staphylococcus aureus. Br J Dermatol 1999; 140: 518-520. 